\title{
The Finite Element Analysis of Hook Based on Workbench
}

\author{
Huang Guojian ${ }^{1, a}$, Liu Jin ${ }^{1, b}$, Chen Min ${ }^{1, c}$ and Peng Qi-feng ${ }^{1, d}$ \\ ${ }^{1}$ Guangzhou Academy of Special Equipment Inspection \& Testing, Guangzhou Guangdong \\ 510663, China \\ aguojian.huang@gmail.com, ${ }^{b}$ liujin8687@qq.com, 'zidane2006@qq.com, d1986554683@qq.com
}

Keywords: Hook; Finite Element; Strength; Fatigue Life

Abstract. In order to improve the safety of the hook, the hook was finite element analyzed. Firstly, the geometric model and finite element model were created. Then the material parameters and boundary conditions were applied to the finite element model, the load was studied specially. Finally, the static strength and fatigue life analysis were carried on. The analysis results indicate that the maximum stress of the hook was $93.5 \mathrm{MPa}$, less than the allowable stress value, the minimum fatigue life was 34 thousand times, and the commuted theoretical life was 6.9 years. This method can reflect the static strength and fatigue life, and provide a reference for improving the safety of the hook.

\section{Introduction}

The hook is an important bearing member and the most widely used extracting device, its performance directly affects the safety of the cranes' operation, once the hook is damaged, it will cause casualties or major economic loss [1]. Given the importance and danger of the hook, many experts and scholars carried on extensive research on its strength, fatigue life and other at work using finite element analysis techniques.

Professor Xuan Fu-zhen carried on finite element analysis of the safety of boiler membrane wall tube screen turning over cliver, the static analysis was carried on firstly, fatigue life was carried on on this basis [2]. Chen Xue-chun carried on FEM analysis of the hot-metal ladle turnover hook intensity [3]. Professor Gao Shun-de carried on simulation research on impaction about hook block of polar crane for nuclear power plant, the result gave some significant theory for the security design of polar crane for nuclear power plant [4]. Yang Chao-li carried on research on the optimization design of roll-over hook by Finite Element Analysis [5]. Ji Wang et al. carried on contrast analysis by finite element for the hook with ladder and T section based on Solidworks [6]. Professor Qiang Bao-min estimated the fatigue life of the hook crossbeam with the linear cumulative damage theory; it could improve the safety of the hook crossbeam [7,8].

On the basis of these theories, taking the hook of a car assembly plant for the study, static strength analysis and fatigue life analysis of the hook was studied.

\section{Creating Model}

Firstly, the geometric model needed to be created in 3D modeling software Solidworks, and then the geometric model was imported to the Workbench to create a finite element model.

Creating the Geometry Model. The accuracy of the geometric model would directly determine the accuracy of the finite element model, so the geometric model needed to maximize reflect the actual situation. When the geometric model was creating by using Solidworks, the basic structure of the hook was needed to be analyzed firstly, and on this basis, the stretch, cut, scan, fillet and other features were selected to create a geometric model [9]. In order to simplify the modeling and be easy to be analyzed, on the basis of the accuracy of the geometric model was met, the geometric model needed to be simplified reasonably, such as the unstressed assistive devices were ignored, small radius chamfers and threaded holes were simplified, differences between the weld and the base metal were ignored.

Creating the Finite Element Model. The geometric model was imported to Workbench, the mesh module was selected, in order to ensure the quality of elements, the hexahedral-dominated meshing 
method was used[10].By the method, the hexahedral elements were used on the surface, and the hexahedral elements were also used in the internal, when the hexahedral elements couldn't be used, the tetrahedral elements were filled. Because the tetrahedral elements were relatively poor, so the method could ensure the quality of the elements. To further improve the calculating accuracy, the transition and other locations of the finite element model of the hook needed to be refined, based on the need, the meshing refinement level could be set as 1 3. Fig.1 was the finite element model of the hook.

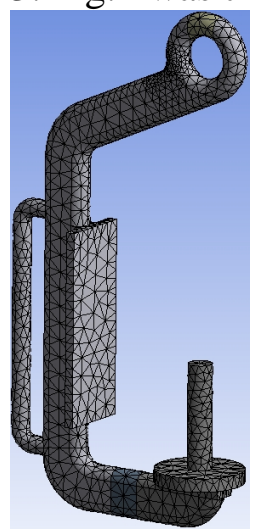

Fig. 1 the Finite Element Model of the Hook

\section{Static Strength Analysis}

Material Parameters. The hook was made of Q235B steel, giving the following specific parameters of the finite element model: (1)elastic modulus $\mathrm{E}=210 \mathrm{GPa}$; (2)density $\rho=7.8 \times 10-6 \mathrm{~kg} / \mathrm{mm}^{3}$; (3)Poisson's ratio $\mu=0.3$; (4)yield strength $\sigma_{\mathrm{s}}=235 \mathrm{MPa}$.

During the static strength analysis, the strength safety factor $n=1.48$ [11], so the allowable stress of the hook was:

$$
[\sigma]=\frac{\sigma_{s}}{n}=158.8 \mathrm{MPa} \text {. }
$$

Boundary Conditions. The boundary conditions included constraints and loads.

The constraints needed to be applied to the finite element model, otherwise the finite element model couldn't be calculated. Meanwhile the constraints should be accuracy and reflected the practical situation, otherwise it would be difficult to get accurate results. In the work condition, the ear was connected with spreader, it could be approximated that is fixedly connected, a fixed constraint was applied to a portion of the lower surface of the ear, as shown in A region of Fig.2.

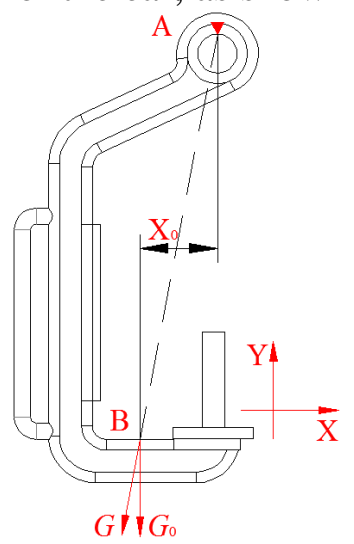

Fig. 2 the Schematic of the Load Direction of the Hook

(Note: Point A is the constrained region; Point B is the loading area, the arrow indicates the direction of the load) 
Since the working conditions were complex, so the most dangerous working conditions should be considered. The load included load region, load direction and load magnitude, the load region located below the hook, as shown in B region of Fig.2.

Fig. 2 was the schematic of the load direction of the hook, for ease of analysis, the constrained region and the loading area were simplified to a point, wherein point A was the constrained region; point B was the loading area. The loads acted on point B were $G_{0}$ and $G$, wherein the direction of $G_{0}$ was Y negative, and the direction of $G$ was from point $\mathrm{A}$ to point $\mathrm{B}, \mathrm{X}_{0}$ was the load eccentricity distance, its value was the distance from point $\mathrm{A}$ to point $\mathrm{B}$ in the $\mathrm{X}$-axis direction. The load direction was easy to apply to Y negative mistakenly, as shown $G_{0}$ in Fig.2. When the load was $G_{0}$, this load would generate bending moment $M_{0}$ at point $\mathrm{A}$, and its value was:

$$
M_{0}=G_{0} \times \mathrm{X}_{0} .
$$

Because of the existence of this bending moment, during the finite element calculation, it would produce big stress at point A. In reality, the hook was not rigidly fixed at work, but could be as slight shifting along with the load, so the load couldn't produce bending moment at point A, it could be seen that the direction of the load was from point A to point B inevitably, as shown $G$ in Fig.2.

In operation, the three hooks suspended to a spreader lifted automotive parts, the maximum lifting load $L L=300 \mathrm{~kg}$, considering the impact of lifting together and dynamic loads, the maximum dynamic load of the lifting system was:

$$
G_{t}=L L \times \mathrm{g} \times \eta_{0} \times \eta_{1} .
$$

Where, $\eta_{0}$ was the lifting together coefficient, its value was $1.1, \eta_{1}$ was the dynamic load coefficient, its value was 1.1[11], g was the gravity acceleration, its value was $9.8 \mathrm{~m} / \mathrm{s}^{2}$.

The lifting system withstood the maximum dynamic load by the three hooks on average, thus according to Eq.3, the load of every hook was:

$$
G=\frac{G_{t}}{3}=\frac{L L \times \mathrm{g} \times \eta_{0} \times \eta_{1}}{3} .
$$

Each parameter was substituted into the Eq.4, then $G=1185.8 \mathrm{~N}$.

The Result of Static Strength Analysis. After the constraints and loads were applied, static strength analysis could be carried out, the analysis results shown in Fig.3.

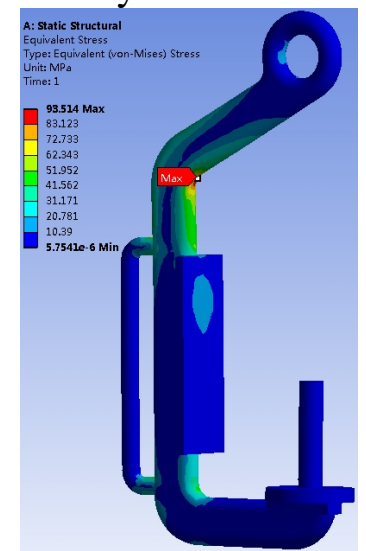

Fig. 3 the Stress Contour of the Hook

Fig.3 showed the maximum stress of the hook was $93.5 \mathrm{MPa}$, located at a turning point, the maximum stress value was less than the allowable stress value $158.8 \mathrm{MPa}$, so the static strength of the hook met the requirements.

But it didn't mean the hook could work safely when the static strength met the requirements, because the hook worked frequently, it was easy to produce fatigue damage at alternating loads, when the damage reached a critical value, the hook would suddenly break, it would cause serious consequences for production, so the fatigue life of the hook would be studied below. 


\section{Fatigue Life Analysis}

The stress spectrum of the dangerous parts was the important basis for the fatigue life prediction; the authenticity of the stress spectrum determined the accuracy of life prediction [12]. It could truly reflect the distribution of the stress spectrum of the hook that the result of static strength analysis was the input of the stress spectrum.

When the fatigue life analysis was performed, the fatigue strength factor $\mathrm{Kf}$ should be set, which was less than 1, for correcting the error between the actual parts (notched) and the SN curve of the experimental work (smooth), the value was set as 0.9 in this analysis [11]. Because the fatigue type was high-cycle fatigue, so the analysis type was set as stress fatigue [13]. The load type was set as symmetrical cyclic load, Goodman theory was selected as the mean stress correction method, stress component was set as equivalent stress [14]. After the analysis parameters were set and fatigue life was analyzed, the fatigue life results could be obtained, Fig. 4 was the result of the fatigue life analysis of the hook.

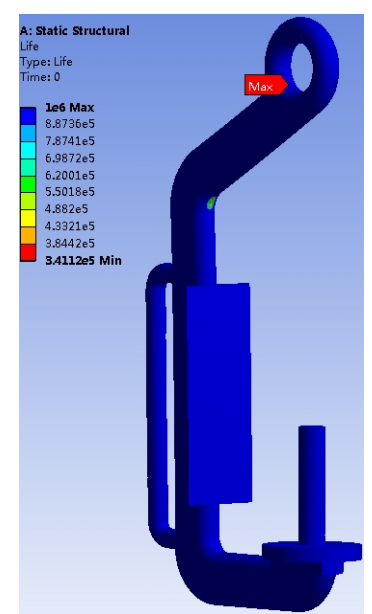

Fig.4 the Result of the Fatigue Life Analysis of the Hook

According to Fig.4, the minimum fatigue life of the hook was 340,000 times. According to the actual use conditions of the hook, the hook was used 350 days per year on average, and 14 hours per day on average, and 10 operating cycles per hour on average, so the operating cycles of the hook was 49,000 times per year. According to the above, the theory life of the hook was $Y=34 / 4.9=6.9$ years.

Also according to Fig.3 and Fig.4, in addition to the fatigue life of a small area of the maximum stress zone was short, the fatigue life of most other regions had reached one million times. It can be seen, in order to improve the fatigue life of the hook, the hook structure, particularly the structure of the maximum stress zone were optimized to improve the fatigue life, so then the safety using time was extended.

\section{Conclusions}

The finite element model of the hook was created firstly, then material parameters and boundary conditions were applied, on the basis, the static strength and fatigue life analysis were carried on, the analysis results was shown that:

1) The maximum stress of the hook was $93.5 \mathrm{MPa}$, less than the allowable stress value $158.8 \mathrm{MPa}$, so the static strength of the hook met the requirements.

2) The minimum fatigue life of the hook was 340,000 times, the operating cycles of the hook was 49,000 times per year, so the theory life of the hook was 6.9 years.

3) In order to improve the fatigue life of the hook, the hook structure, particularly the structure of the maximum stress zone were optimized to improve the fatigue life, so then the safety using time was extended. 


\section{Acknowledgements}

This work was financially supported by Technology Projects of Guangdong Provincial Quality and Technical Supervision (2014PT02), Technology Projects of State Administration of Work Safety (Guangdong- 0012-2015AQ), Guangzhou Innovation Platform and Sharing Special Projects (201509010008), Pearl River Technology Nova Special Projects (2013075), Technology Projects of Special Equipment Technology Collaboration Platform (2014SEK003、2014SEK002 、 2014SEK001).

\section{References}

[1] Bai Xue-yong, Li Shu, Li Yong-gang. Stress Analysis of Lifting-hoot with ANSYS Software [J]. Coal Mine Machinery,2009,11:86-87.

[2] Hua Qing. Finite Element Analysis of the Safety of Boiler Membrane Wall Tube Screen Turning Over Cliver [D]. East China University of Science and Technology,2013.

[3] Chen Xue-chun. FEM Analysis of the Hot-metal Ladle Turnover Hook Intensity[J].Metallurgical Equipment, 2009,S3:9-11+94.

[4] Li Zhang-lei. Simulation and Research on Impaction about Hook Block of Polar Crane for Nuclear Power Plant [D]. Dalian University of Technology,2012.

[5] Yang Chao-li. Research on the Optimization Design of Roll-over Hook by Finite Element Analysis [J]. Journal of Kunming University,2008,04:81-85.

[6] Ji Hong; Qin Chang-wei; Yang Xu. Contrast Analysis by Finite Element for the Hook with Ladder and $\mathrm{T}$ Section based on Solidworks [J]. Journal of Liaoning Institute of Science and Technology,2012,03:18-20.

[7] Qiang Bao-min, Yuan Ren-wu, Zhang De-kuan, Wei Xing. The Fatigue Life Estimation of the Bridge Crane's Hook Crossbeam [J]. Science Technology and Engineering, 2015,01:224-227+236.

[8] Yang Bi-gen, Qiang Bao-min, Yuan Ren-wu, Wei Yun-ping. The Residual Fatigue Life Prediction of Crane Hook Crossbeam [J]. Science Technology and Engineering, 2015,11:196-200.

[9] Li Jia-wei. Design Method of Automotive Drilling Big Derrick Based on Solidworks [J]. Journal of Huaihai Institute of Technology(Natural Science Edition),2015,03:11-14.

[10] Song Yuan-ling, Wei Liang-bao, Hou Ming-kai. Research on the Optimization Design of the Main Girder of the Bridge Crane Based on Workbench [J]. Hoisting and Conveying Machinery,2015,09:32-35.

[11] GB/T 13752-92, Design Rules for Cranes. Beijing, China Standard Press,1993:16-17.

[12] Christopher S Grimmer, C K H Dharan. High-cycle Fatigue Life Extension of Glass Fiber/Polymer Composites with Carbon Nanotubes [J]. Composites Science and Technology, 2010, 70(6): 901-908.

[13] Chul-Hee JO, Sung-Woo IM, Won-Chul CHO, Kwan-Kyu Park. Fatigue crack in large-scale tubular joints for offshore structures [J].Science China Technological Sciences, 2011, 54 (3) : 705-714.

[14] JONES R, MOLENT L, KRISHNAPILAI K. An equivalent block method for computing fatigue crack growth [J]. International Journal of Fatigue, 2008, 30(9): 1529-1542. 